\title{
Preparation and Characterization of Peg-Albumin-Paclitaxel (PAP) Nanoparticles Intended to Treat Breast Cancer
}

\author{
Ramchander Tadakapally ${ }^{1}$, Mohammad Habbibudin ${ }^{2}$, Jithan Aukunuru ${ }^{3,4, *}$ \\ ${ }^{1}$ Mother Teresa College of Pharmacy, Ghatkesar, Hyderabad, India \\ ${ }^{2}$ Shadan College of Pharmacy, Peerancheru, Hyderabad, India. \\ ${ }^{3}$ Mankind Research Center, Manesar, Haryana, India \\ ${ }^{4}$ Technology Consultants, Hyderabad, India.
}

\begin{abstract}
The aim of the present research work is to prepare serum stable long circulating PEGylated paclitaxel-BSA nanoparticles for breast cancer and were prepared by desolvation technique. Prepared nanoparticles were characterized for drug entrapment efficiency (EE), particle size, scanning electron microscope (SEM), zeta potential, fourier transform infrared spectroscopy (FTIR) and differential scanning calorimetry (DSC). In-vitro release studies were performed in phosphate buffer saline $\mathrm{pH} 7.4$ at $37^{\circ} \pm 0.5^{\circ} \mathrm{C}$ for 14 days. The in-vivo animal studies were performed for drug release studies for 21 days and cell viable assay was performed. The entrapment efficiency was about $90.5 \%$, the mean particle size of obtained nanoparticles was $150-400 \mathrm{~nm}$ and was apparently spherical in shape, with smooth surface. The zeta potential is found to be $-29 \mathrm{mV}$ and the nanoparticles are found to be stable. Drugexcipient interaction studies were conducted by FT-IR studies, it demonstrated that the drug was not changed in the formulation during the fabrication process. The DSC results obtained also showed no significant shift in the endothermic peaks confirming the stability of the drug in the formulations and polymeric peaks revealed that drug is in amorphous state in the formulations. The paclitaxel-BSA nanoparticles exhibit a most interesting release profile with small initial burst followed by slower and controlled release.
\end{abstract}

Keywords : Paclitaxel, albumin, long circulating polymeric nanoparticles, PEGylation, desolvation, breast cancer.

\section{Introduction:}

Novel/Controlled drug delivery technology represents one of the broader areas of science, which involves multidisciplinary scientific approach, contributing to human health care and pharmaceutical development ${ }^{1}$. Drug targeting is defined as a selective drug release at specific physiological sites, cells, tissues, organs or in which the pharmacological effect is required. The concept of drug targeting is used in attempts to improve the therapeutic index and efficacy of drugs by increasing their localization to specific organs, tissues or cells and by decreasing their potential toxic, side and adverse effects at normal sensitive sites ${ }^{2-3}$. The efficacy of many drugs is often limited by their potential to reach the site of therapeutic action ${ }^{4}$. In most cases (conventional dosage forms), only a small amount of administered dose reaches the target site, while the 
majority of the drug distributes throughout the rest of the body in accordance with its physicochemical and biochemical properties ${ }^{3,5}$. Therefore, developing a drug delivery system, that optimizes the pharmaceutical action of a drug, while reducing its toxic side effects in vivo is a challenging task. Chemotherapy has become an integral component of cancer treatment for most cancers. Conventional chemotherapeutic agents still exhibit poor specificity in reaching tumor tissue and are often restricted by dose-limiting toxicity. ${ }^{6}$ The combination of developing controlled release and targeted drug delivery may provide a more efficient and less harmful solution to overcome the limitations found in conventional chemotherapy. The efficacy of cancerous chemotherapy is often limited by serious side effects because of the toxicity of anticancer drugs to both tumor and normal cells ${ }^{7,8}$. In targeted cancer therapy, conventional chemotherapeutic agents, which lack intrinsic target specificity, are rationally modified to focus and redirect their cytotoxicity to tumor cells. The development of a drug delivery system faces several challenges: reaching the target site, which is often, far away from the administration site (drug targeting), remaining at the target site to deliver the drug, preferably in a time controlled manner, limiting the drug's adverse effects and ensuring biocompatibility ${ }^{9}$. The need for intravenous (IV) formulations and the advantage of enlarging surface contact with an external medium to control release kinetics have encouraged the development of nanoparticles ${ }^{10}$. Despite several advancements, the drug transport at high concentrations to solid tumors seems still to be a challenge; nanoparticles have been widely attempted for delivering cancer agents to tumors. Sanjeeb K shaoo et al., 2011 prepared polymeric nanoparticles and used as carriers for systemic and targeted drug delivery systems ${ }^{11}$. Paclitaxel (PTX) $\left(\mathrm{C}_{47} \mathrm{H}_{51} \mathrm{NO}_{14}\right)$, a poly-oxygenated naturally occurring di-terpenoid isolated from the bark of the Pacific yew tree, Taxus brevifolia. It was first discovered by Dr.Monroe E.Wall and Mansukh C.Wani and its melting point is $216-217^{\circ} \mathrm{C}$, white crystalline powder. Taxanes had a profound impact on variety of malignancies like ovarian cancer, breast cancer, lung cancer prostate cancer, head and neck tumours, kaposis sarcoma and urological Malignances. PTX acts during the mitotic phase of cell division causes microtubule solubilizing agent. Inhibits angiogenesis related cell migration, proliferation and collagenase secretion by these properties, it acts as anti-neoplastic agent ${ }^{12}$ However PTX has limitations it is highly lipophilic and poorly water soluble drug solubility is less than $0.01 \mathrm{mg} / \mathrm{ml}$. It's always associated with toxic side-effects. It should be only used when the potential benefits of paclitaxel therapy outweigh the possible risks. Because of its hydrophobic nature the current clinical dosage form of paclitaxel consists of a 1:1 (v/v) mixture of ethanol and Cremophor EL, a non-ionic polyethoxylated castor oil solubilizer, diluted in water for injection prior to infusion. This pharmaceutical formulation; however, is associated with a number of concerns including stability, filtering requirements and use of non- plasticized containers. Moreover, some of the side-effects such as severe hypersensitivity reactions. ${ }^{9,10}$ The multiple dosing of the drug, required to maintain the therapeutic drug concentration in the tumour, causes nonspecific toxicity such as nephrotoxicity, neurotoxicity and cardio toxicity in patients in addition to the adverse effects of taxol including neutropenia, peripheral neuropathy, arthralgia, myalgia, mucositis, nausea, vomiting and alopecia. The vehicle has been shown to cause fatal hypersensitivity reactions at nearly every step in the development path, in both preclinical and clinical testing. ${ }^{11-12 .}$ To overcome these problems Nanoparticle Albumin-bound (nab) technology which has been developed by Abraxis biosicences is a novel patented technology. Albumin is versataile protein carrier for drug delivery, it is non toxic, bio-compatible, non immunogenic and bio degradable. It is ideal material to achieve safe, solvent free, efficient and targeted drug delivery the first formulation entered the market and is Abraxane. K. Madhavi and Jithan $e$ tal., 2011 prepared polymeric based polymeric based nanoparticles using bovine-serum albumin. In this study, we particularly focused on pegylated albumin nanoparticles. PEG is used to impart the in-vivo longevity to drug carriers, PEGylation reduces the protein binding (opsonization) stealth nanoparticles circulates longer time in the blood it leads to more accumulation in the tumour, interacts more with target and enhances tumour targeting. Mittsching B $e$ tal, 2011 administered albumin bound (nab) paclitaxel nanoparticles as intravenous infusion. ${ }^{\text {14. }}$ In this study, we further aimed at improvement of the formulation by conjugating pegylation to nab technology. The main objective of this study was to prepare and evaluate serum stable long circulating PEG-AlbuminPaclitaxel nanoparticles intended to be administered in brest cancer, improving the therapeutic index of the drug by increasing its solubility and tumour accumulation.

\section{Materials and Methods:}

Paclitaxel was purchased from Natco pharmaceuticals Ltd. Hyderabad. Bovine serum albumin [fraction v] specification - Albumin min 98.5\%, was purchased from Qualikems fine chem. Pvt. Ltd, Mumbai. Glutaraldehyde was purchased from SDFCL, Hyderabad. Methanol was purchased from SDFCL. Methanol HPLC grade was purchased from SDFCL, Hyderabad. Ethyl acetate was purchased from Qualikems Fine chem. 
All the other chemicals were of analytical grade. Magnetic stirrer, cyclomixer, ultracentrifuge and micro centrifuge bought from REMI Equipments Pvt. Ltd. were used. HPLC from waters, used in the analysis of drug levels in the plasma and the tissues. A Shimadzu UV-Visible spectrophotometer was used in the analysis of samples from in vitro drug release and drug content assays. A bath sonicator from Fischer brand was used.

\section{De-solvation technique:}

Paclitaxel nanoparticles were prepared by using desolvation technique. Nanoparticles of paclitaxel were prepared by using biodegradable bovine serum albumin as the polymer. This experiment was conducted in dark condition as the drug degrades in the presence of light. Polymeric solution: BSA was taken between 50mg and $200 \mathrm{mg}$ and was dissolved in $2 \mathrm{ml}$ of purified distilled water.

\section{Drug solution:}

Weigh paclitaxel and dissolve in $8 \mathrm{ml}$ of ethanol .Drug solution is added drop-wise to the above polymeric solution under magnetic stirring $(500 \mathrm{rpm})$ which results in the formation of opalescent suspension at room temperature. After desolvation, $0.11 \mathrm{ml}$ of $8 \%$ gluteraldehyde in water $(\mathrm{V} / \mathrm{V})$ was added to the suspension. This cross linking process of the colloidal suspension was performed over a time period of $24 \mathrm{hrs}$ as the composition given in table 1 . The suspension then obtained was subjected to 5 cycles of differential centrifugation $(12,000 * \mathrm{~g}, 8 \mathrm{~min})$. The pellets then obtained by centrifugrsed to original volume with distilled water. Each redispersion step was performed in a bath sonicator over $10 \mathrm{~min}^{15}$

Table: 01 preparation of serum stable long circulating polymeric nanoparticles of paclitaxel nanoparticles

\begin{tabular}{|c|c|c|c|c|c|}
\hline FORMULATION & $\mathbf{F}_{1}$ & $\mathbf{F}_{2}$ & $\mathbf{F}_{3}$ & $\mathbf{F}_{4}$ & $\mathbf{F}_{5}$ \\
\hline Paclitaxel & $100 \mathrm{mg}$ & $100 \mathrm{mg}$ & $100 \mathrm{mg}$ & $100 \mathrm{mg}$ & $100 \mathrm{mg}$ \\
\hline BSA & $200 \mathrm{mg}$ & $200 \mathrm{mg}$ & $200 \mathrm{mg}$ & $200 \mathrm{mg}$ & $100 \mathrm{mg}$ \\
\hline PEG(6000) & ---------- & $20 \mathrm{mg}$ & ----------- & ---------- & --------- \\
\hline PEG(4000) & --------- & ---------- & $20 \mathrm{mg}$ & --------- & --------- \\
\hline PEG(1500) & ----------- & ----------- & ----------- & $20 \mathrm{mg}$ & --------- \\
\hline $\begin{array}{l}\text { Glutaraldehyde } \\
(8 \% \text { v/v) }\end{array}$ & $0.11 \mathrm{ml}$ & $0.11 \mathrm{ml}$ & $0.11 \mathrm{ml}$ & $0.11 \mathrm{ml}$ & $0.11 \mathrm{ml}$ \\
\hline Ethanol & $8 \mathrm{ml}$ & $8 \mathrm{ml}$ & $8 \mathrm{ml}$ & $8 \mathrm{ml}$ & $8 \mathrm{ml}$ \\
\hline Distilled water & $2 \mathrm{ml}$ & $2 \mathrm{ml}$ & $2 \mathrm{ml}$ & $2 \mathrm{ml}$ & $2 \mathrm{ml}$ \\
\hline
\end{tabular}

\section{PEGylation of paclitaxel- bovine serum albumin nanoparticle:}

Prepared BSA-paclitaxel nanoparticle were stirred with PEG $(6000,4000,1500)$ respectively for at least $5 \mathrm{~min}$ in probe sonicator. The resultant solution was taken in a RBF and lyophilized under freeze dryer. It takes approximately $2 \mathrm{hrs}$ for freezing and $1 \mathrm{hr}$ for drying under vacuum in freeze dryer. The resultant dried particles obtained were collected as PEGylated BSA paclitaxel nanoparticle for further use. The prepared nanoparticles using this technique were characterized by a scanning electron microscope and a zeta-sizer. In formulation $\mathrm{F}_{2}, \mathrm{~F}_{3}$, and $\mathrm{F}_{4}$ PEG 6000, PEG 4000, and PEG 1500 are used respectively for the PEGylation of the BSA- Paclitaxel nanoparticles. PEGlyation of the BSA- Paclitaxel nanoparticles is done to prepare serum stable long circulating nanoparticles in the blood.

\section{Characterization of PEG-Albumin-Paclitaxel Nanoparticles:}

\section{Particle size, Zeta Potential and surface morphology:}

In order to examine the particle surface morphology and shape, scanning electron microscopy (SEM) was used. A paclitaxel nanoparticles solution was spread over a slab and dried under vacuum. All the samples such as 1) Paclitaxel-BSA nanoparticles, 2) Paclitaxel-BSA+PEG4000 nanoparticles, 3) PaclitaxelBSA+PEG1500 nanoparticles were shadowed in a cathodic evaporator with gold layer 20nm thick photograph were taken using a JSM-5200 SEM (Toky, Japan) operated at 20Kv. The electrophoretic mobility and zeta 
potential were measured using a zeta potentiometer (Malvern Zeta sizer). To determine the zeta potential, nanoparticles sample were diluted with $\mathrm{KCl}(0.1 \mathrm{~mm})$ and placed in the electrophoretic cell where an electric field of $15.2 \mathrm{~V} / \mathrm{cm}$ was applied. Each sample was analyzed in triplicate.

\section{Drug entrapment efficiency:}

Drug entrapment efficiency was determined by the dialysis method and was calculated for the samples of Paclitaxel-BSA nanoparticles such as Paclitaxel-BSA+PEG6000 nanoparticles, Paclitaxel-BSA+PEG4000 nanoparticles, Paclitaxel--BSA+PEG1500 nanoparticles Take a beaker containing 100ml of phosphate buffer of pH 7.4 and keep on the magnetic stirrer for stirring. 10mg of samples was weighed and taken in a broken test tube containing $10 \mathrm{ml}$ of phosphate buffer of $\mathrm{pH} 7.4$. Bottom end of the test tube was packed with the diffusion membrane. Place the test tube inside the beaker and adjust it such that the test tube is submerged in the beaker containing phosphate buffer of $\mathrm{pH}$ 7.4. Now collect the samples $10 \mathrm{ml}$ from the beaker for every $1 \mathrm{hr}$ till $10 \mathrm{hrs}$ and replace it with the fresh medium of buffer.

Entrapment efficiency $\% \quad=$ Weight of the drug in nanoparticles $\times 100 \%$

Weight of the nanoparticles

\section{Drug-excipient compatibility studies (FT-IR studies):}

FT-IR is used for the drug-excipient compatibility samples of about 5mg was mixed thoroughly with100mg KBr IR powder and compacted under vacuum at a pressure of about 12psi, for $3 \mathrm{~min}$. The resultant disc was mounted in a suitable holder in Perkin Elmer IR spectrophotometer and the IR spectrum was recorded from $4000 \mathrm{~cm}^{-1}$ to $400 \mathrm{~cm}^{-1}$ in a scan time of $12 \mathrm{~min}$. these studies were done for 1) Bovine serum albumin 2) PEG 6000 3)PEG 4000 4) PEG 1500 5) Pure drug (paclitaxel) 6) Paclitaxel-BSA nanoparticles 7) PaclitaxelBSA+PEG6000 nanoparticles 8) Paclitaxel-BSA+PEG4000 nanoparticles 9) Palitaxel-BSA+1500 nanoparticles.

\section{Differential scanning calorimetry (DSC):}

Differential scanning calorimetry (DSC) is a thermo analytical method used to study thermal transitions involving energy or heat capacity changes. Thermal properties of the powder samples were investigated with differential scanning colorimetry. Approximately $10 \mathrm{mg}$ of samples was analyzed in an open aluminum pan and heated at scanning rate of $10^{\circ} \mathrm{c} / \mathrm{min}$ between $0^{\circ} \mathrm{C}$ and $400^{\circ} \mathrm{C}$. Magnesia was used as the standard reference materials. The thermograms of paclitaxel were obtained for the samples paclitaxel-BSA nanoparticles, paclitaxel-BSA+PEG6000 nanoparticles, paclitaxel-BSA+PEG4000 nanoparticles, paclitaxel-BSA+PEG1500 nanoparticles.

\section{In-vitro drug release studies:}

In-vitro drug release experiments were conducted in dark conditions as the drug paclitaxel degrades in the presence of light. The release medium used was phosphate buffer of $\mathrm{pH} 7.4$ which contains ascorbic acid and butylated hydroxytoulene at a concentration of $1 \%$ and $0.1 \%$ respectively to prevent degradation. About 10mg of each sample paclitaxel-BSA nanoparticles, paclitaxel-BSA+PEG6000 nanoparticles, paclitaxelBSA+PEG4000 nanoparticles, paclitaxel-BSA+PEG1500 nanoparticles were weighed and redispersed in 100ml of release medium (phosphate buffer $\mathrm{pH} 7.4$ )The entire beaker was kept at $37 \pm 0.5^{\circ} \mathrm{C}$ under stirring at 100rpm.At the time intervals of $[1,2,3,4,5,6,7,8,9,10,24,48$ till 10days] the samples $10 \mathrm{ml}$ was withdrawn and was replaced with the fresh medium of buffer. The samples withdrawn were filtered by using $0.2 \mu$ sterile filter.

The amount of drug in the release medium was determined by UV-Visible spectrophotometer at 225 $\mathrm{nm}$. The cumulative amount of drug release over the time period was plotted. The data was fitted to various models that indicate the type of drug release. To determine the enhancement in the solubility with the formulation, solubility studies with Paclitaxel and with the formulations were conducted ${ }^{14}$.

\section{In-vivo animal studies:}

Animal experiments were conducted with the institutional animal ethics committee of Geetanjali college of pharmacy, Hyderabad (IAEC No.1684/PO/a/12/CPCSEA). The study was conducted using 12 wistar 
rats divided into 3 groups $(n=4)$, each mice was injected via lateral vein, native Abraxen is injected for group 1,paclitaxel nanoparticle is injected for group 2 , pegylated paclitaxel nanoparticles is injected for group 3, blood sample was collected from retro orbital plexus at different time intervals. Serum was separated, the concentration of paclitaxel was determined by HPLC analysis (Maiti k et al.,int j Pharma 2007; 330(1-2),155$63)$.

Table: 02 Drug entrapment efficiency of paclitaxel nanoparticle formulations

\begin{tabular}{|l|l|l|}
\hline S. No & Formulations & \% Drug Encapsulated \\
\hline 1 & F $_{1}$ & 90.5 \\
\hline 2 & F $_{2}$ & 78.5 \\
\hline 3 & F $_{3}$ & 81.3 \\
\hline 4 & F $_{4}$ & 89.5 \\
\hline
\end{tabular}

\section{Results and Discussions:}

Preformulation studies for paclitaxel have been performed to know the drugs physicochemical properties so as to design it to a suitable formulation. Paclitaxel is a class IV drug classified under BCS classification with low solubility and high permeability. The physicochemical properties were described. Poor solubility leads to poor dissolution, therefore to enhance the dissolution of the drug, different techniques have been employed such as particle size reduction by forming nanoparticles which is a novel technique. SEM was used to determine the particle size of samples such as 1) Paclitaxel-BSA nanoparticles, 2) PaclitaxelBSA+PEG4000 nanoparticles, 3) Paclitaxel-BSA+PEG1500 nanoparticles from fig. 1 and 2, it was concluded that the average particle size for all formulations was found to be in nano range of $150 \mathrm{~nm}-400 \mathrm{~nm}$. Surface morphology and shape were visualized. The particles were appeared as spheres. From this study it has been concluded that there is a size reduction of particle which results in enhancing dissolution rate of the paclitaxel. The results of percentage drug entrapment efficiency are shown in the table. 2. From the results shown in the table, it can be inferred that there is a proper distribution of PTX in the nanoparticles. The percentage entrapment efficiency was found to be $78.5 \%$ to $90.5 \%$. A maximum of $90.5 \%$ drug entrapment efficiency was obtained in the PTX -BSA nanoparticle. Release of the drug from the formulations is about $9.5 \%, 21.5 \%, 18.7 \%$, $10.5 \%$ in different formulations $\mathrm{F}_{1}, \mathrm{~F}_{2}, \mathrm{~F}_{3}, \mathrm{~F}_{4}$ formulations with-in $2 \mathrm{hrs}$ was observed. The initial burst release might be due to the dissociation of surface absorbed drug present in the polymeric matrix. The sustained release activity of the drug was due to the slow release of drug entrapped inside the PEGylated polymeric matrix. Drug compatibility studies were performed by foureier transform infrared spectroscopy (FT-IR) has been performed to know the drug excipient compatibility and to check the presence of drug and polymer in the nanoparticle formulations. The chemical interaction between the drug and the polymer can be observed by the change in the infrared profile of nanoparticles of paclitaxel and can be interpreted by careful study of spectra from fig. 3 to 11. FT-IR is done for 1) Bovine serum albumin 2) PEG 6000 3) PEG 4000 4) PEG 1500 5) pure drug (paclitaxel) 6) Paclitaxel-BSA nanoparticles 7)Paclitaxel-BSA+PEG6000 nanoparticles 8) PaclitaxelBSA+PEG4000 nanoparticles 9) Paclitaxel-BSA+1500 nanoparticles. FT-IR spectra obtained for paclitaxel shows characteristic peaks at $1732 \mathrm{~cm}^{-1}$ (ketone group, $\mathrm{C}=\mathrm{O}$ stretching vibrations are seen at $1732 \mathrm{~cm}^{-1}$ ), $3481 \mathrm{~cm}^{-1}\left(\mathrm{OH}\right.$ group, broad), $1647.26 \mathrm{~cm}^{-1}$ (amide group, $\left.\mathrm{CONH} 2\right), 2945 \mathrm{~cm}^{-1}\left(\mathrm{C}-\mathrm{H}\right.$ aromatic ring), $1072 \mathrm{~cm}^{-1}(\mathrm{C}-\mathrm{O}$ group), $1244 \mathrm{~cm}^{-1}$. The spectra of paclitaxel loaded polymer blend were not characteristically different from the spectra of the paclitaxel. The peaks appearing at $3481 \mathrm{~cm}^{-1}, 2945 \mathrm{~cm}^{-1}, 1732 \mathrm{~cm}^{-1}, 1558 \mathrm{~cm}^{-1}, 1647.26 \mathrm{~cm}^{-1}$, $1244 \mathrm{~cm}^{-1}, 1111.03 \mathrm{~cm}^{-1}, 1109 \mathrm{~cm}^{-1}, 1072 \mathrm{~cm}^{-1}$ for paclitaxel and their polymers were also appearing in paclitaxelloaded polymer blend, indicating the chemical stability of paclitaxel in the blend. The blends retained the integrity of drug and as a reason these polymers were selected for further studies. FT-IR studies demonstrated that the drug was not changed in the formulation during the fabrication process. DSC is done for the stability test for pure drug and samples, paclitaxel-BSA nanoparticles, paclitaxel -BSA+4000, paclitaxel -BSA+1500. DSC is done for the pure drug and samples, paclitaxel -BSA nanoparticles, paclitaxel -BSA+4000, paclitaxel BSA+1500. The DSC thermograms obtained for the pure drug and for the formulation shown in the fig 12 indicated that there is no significant shift in the endothermic peaks confirming the stability of the drug in the formulations and only polymer peak was observed, which revealed that drug is in amorphous state in the formulations. In-vitro drug release studies of paclitaxel nanoparticles were performed under magnetic stirring for a month under dark conditions. The calibration curve of the drug was constructed to determine the concentration of the drug from the absorbance value. From this the \% drug release was determined. The plots of 
cumulative percentage drug release $\mathrm{v} / \mathrm{s}$ time for all the four formulations $\left(\mathrm{F}_{1}, \mathrm{~F}_{2}, \mathrm{~F}_{3}, \mathrm{~F}_{4}\right.$ and $\left.\mathrm{F}_{5}\right)$ were performed and optimized formulations were compared with pure drug and represented graphically as shown in the fig 13 . In-vivo studies were performed and results were compared with pure drug and represented graphically as shown in the fig 14 where the pegylated albumin naoparticles have more sustained release profile compared to pure drug.
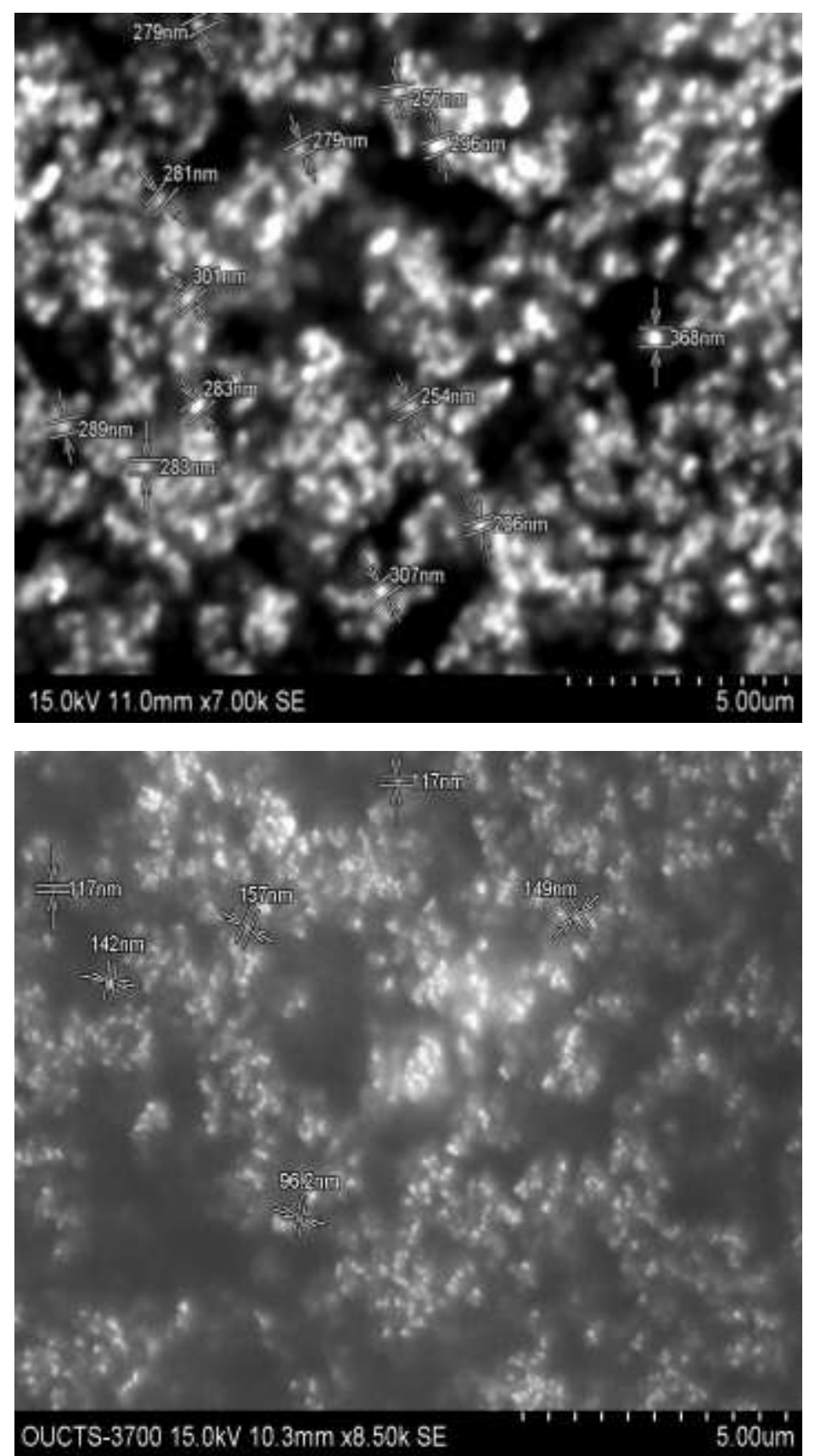

Figure no: 01, 02: SEM pictures of paclitaxel nanoparticles. 


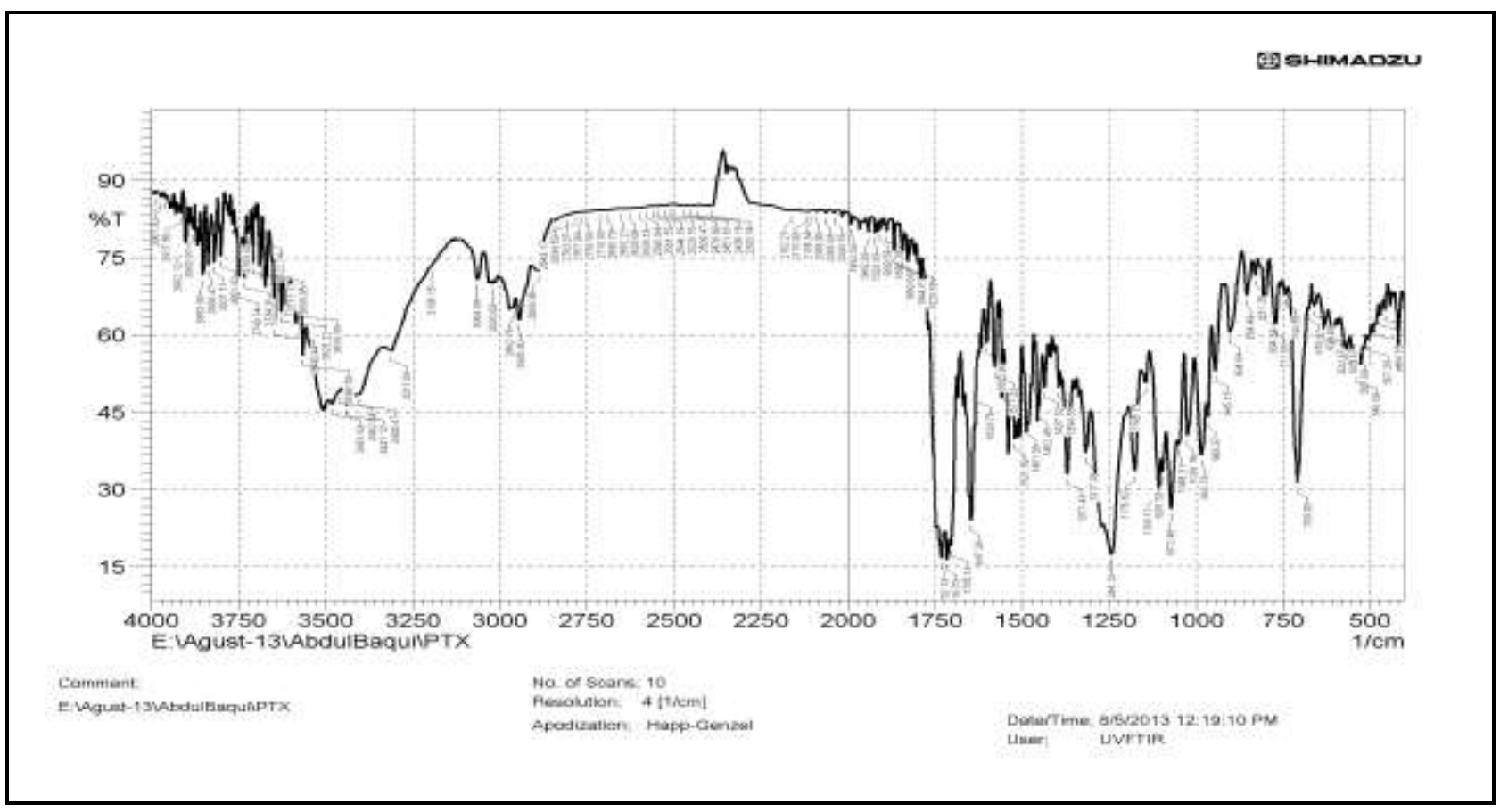

Figure no: 03 FT-IR graph of Paclitaxel

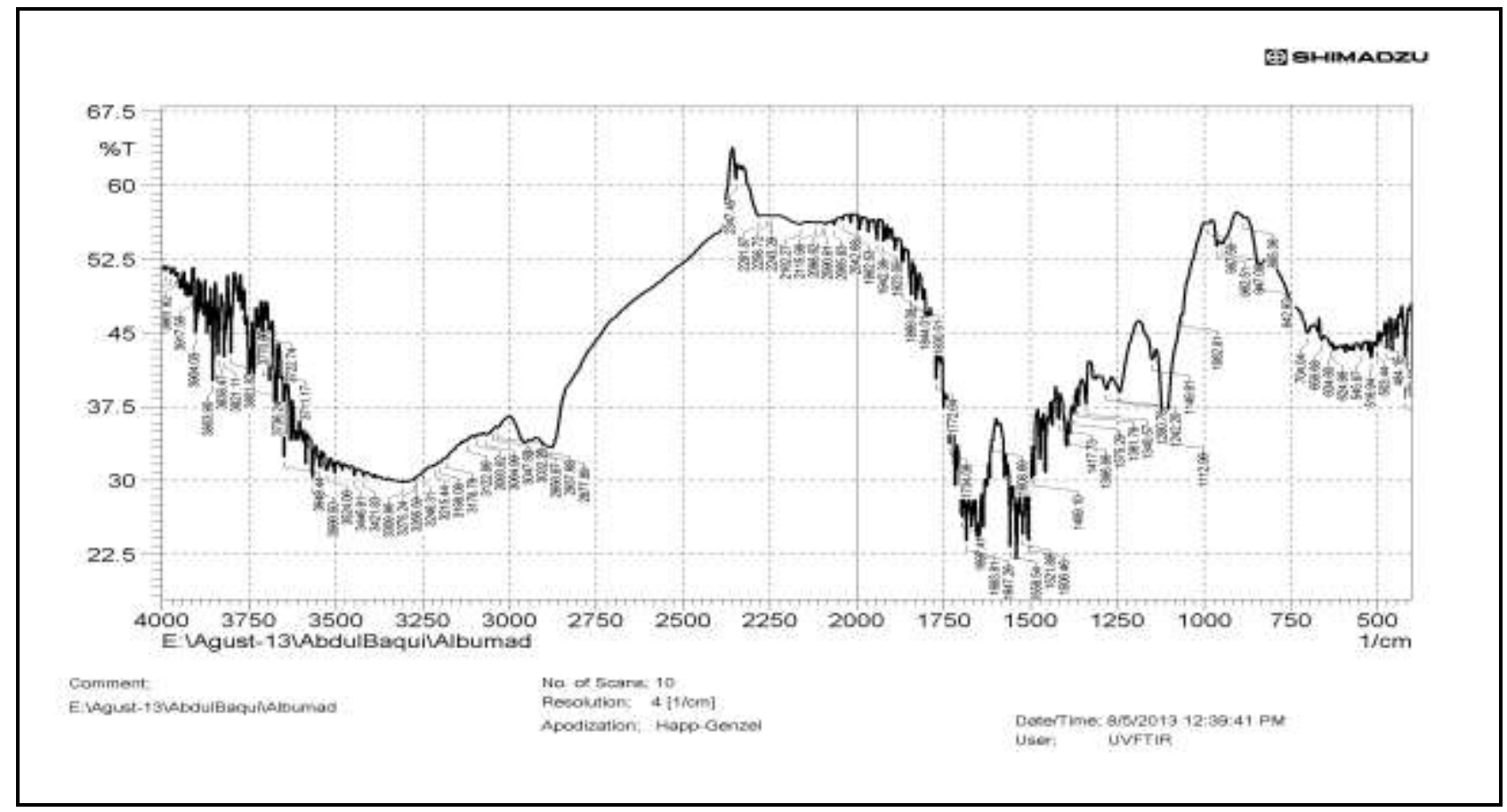

Figure no: 04 FT-IR graph of Bovine serum albumin 


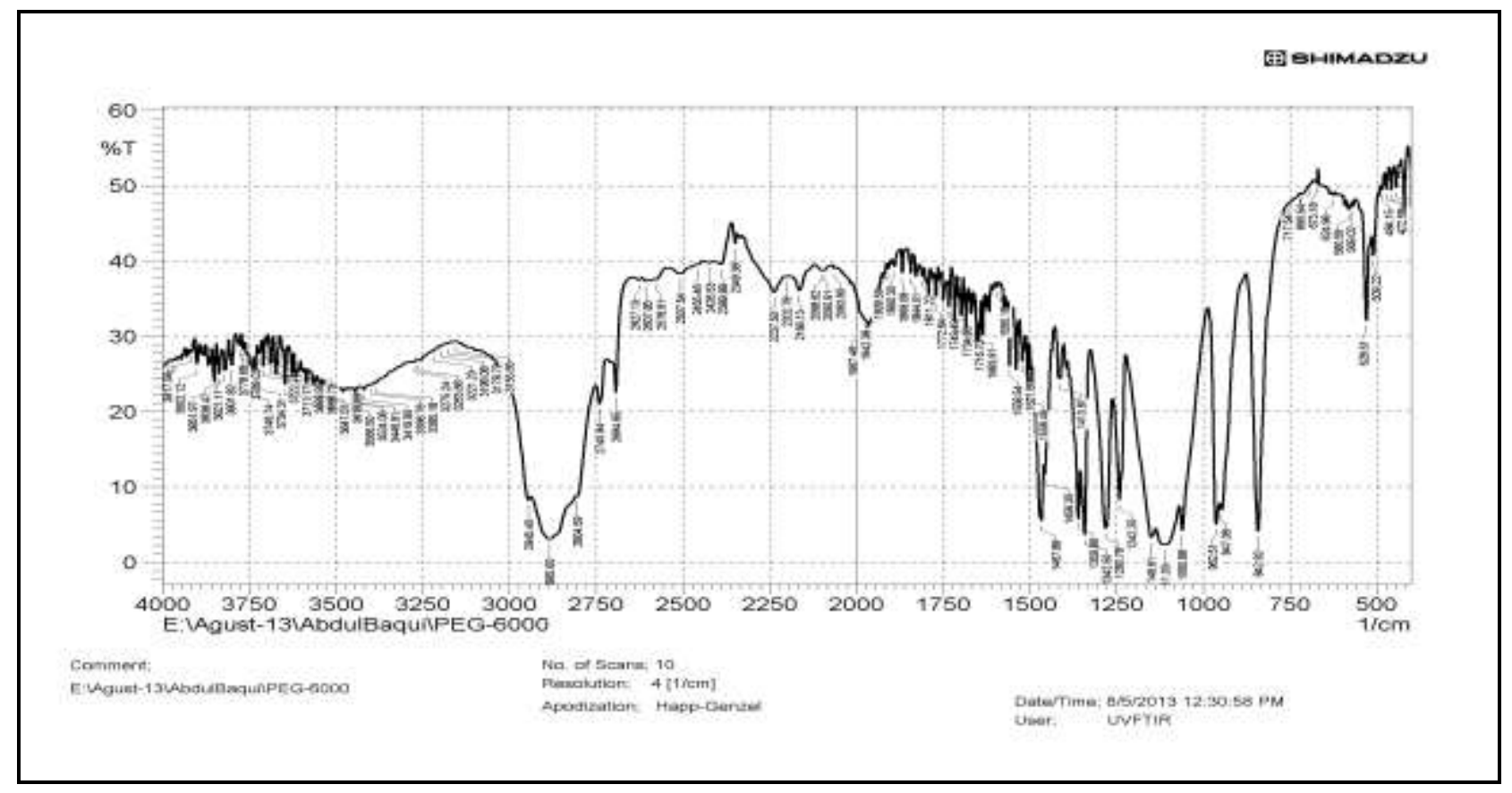

Figure no: 05 FT-IR graph of PEG 6000

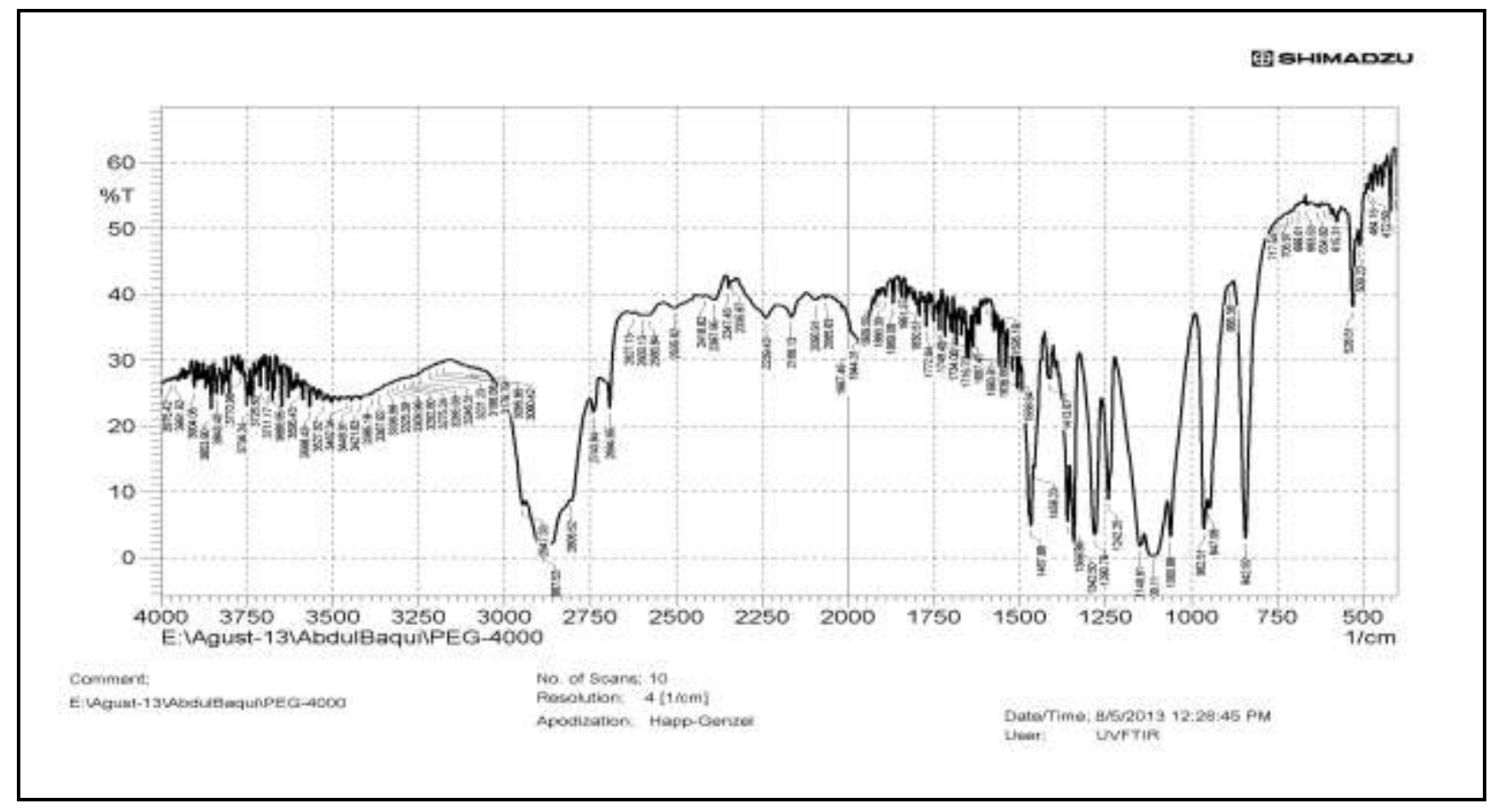

Figure no: 06 FT-IR graph of PEG4000 


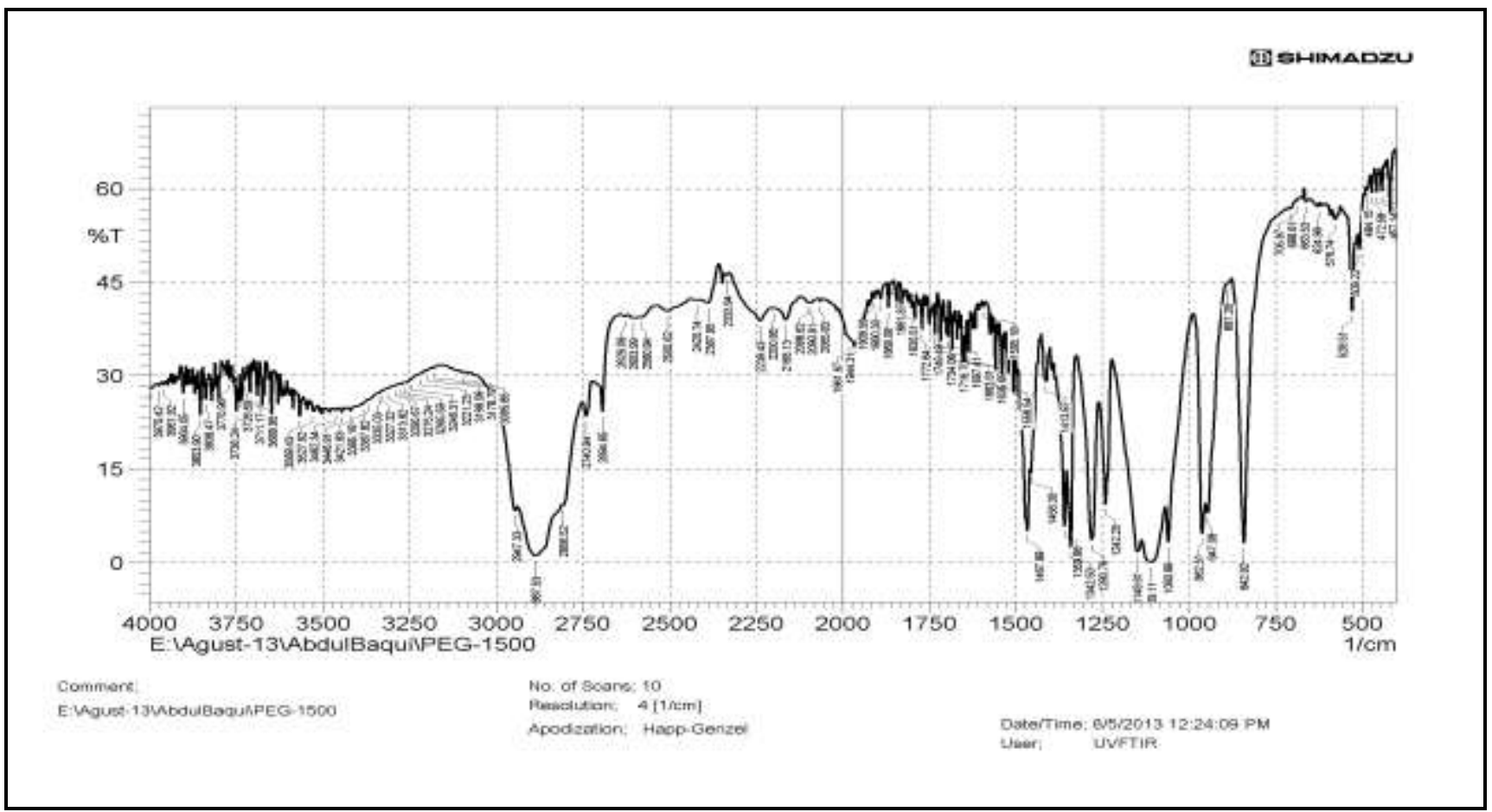

Figure no: 07 FT-IR graph of PEG1500

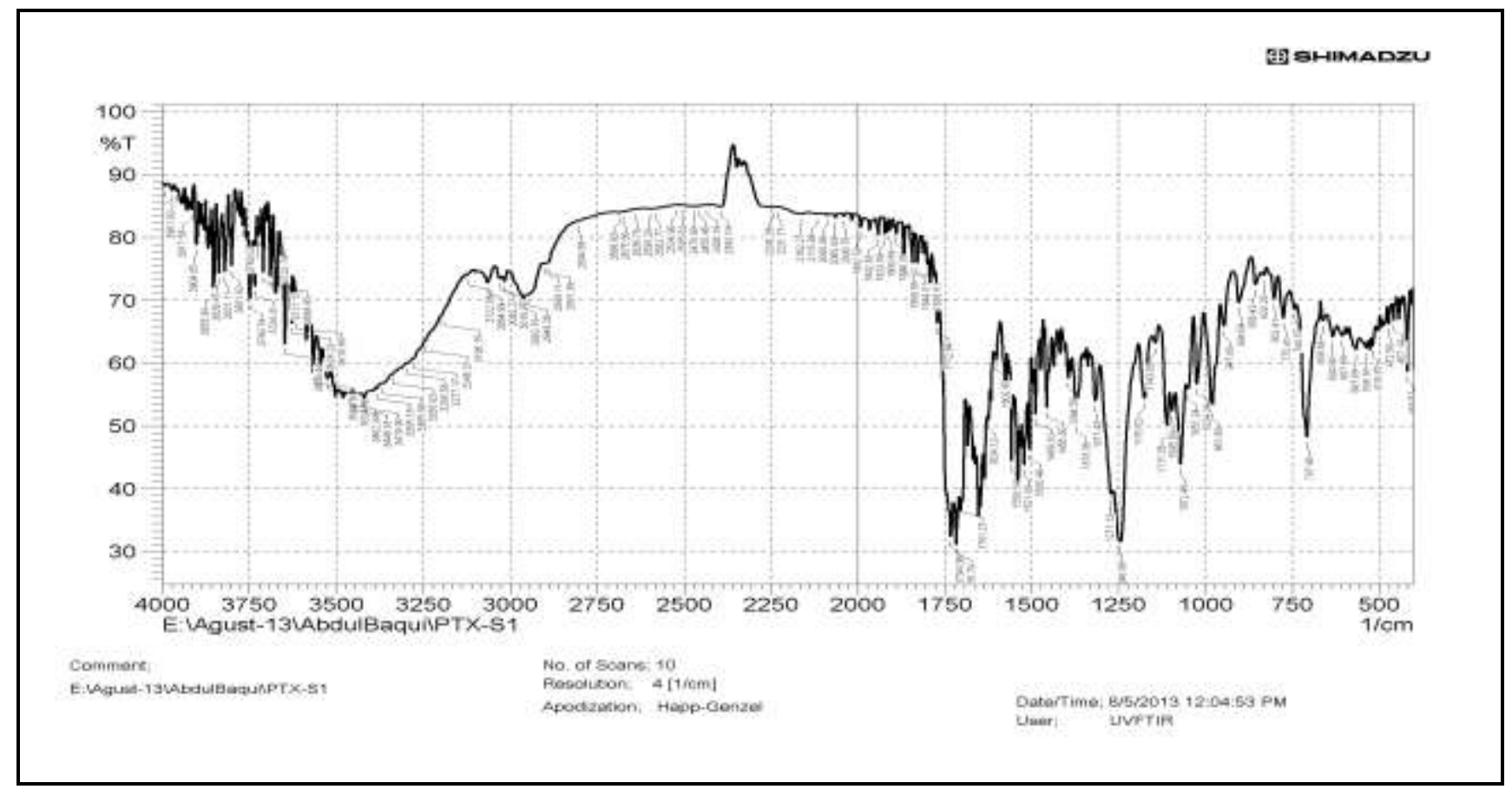

Figure no: 08 FT-IR graph of Paclitaxel-BSA Nanoparticles 


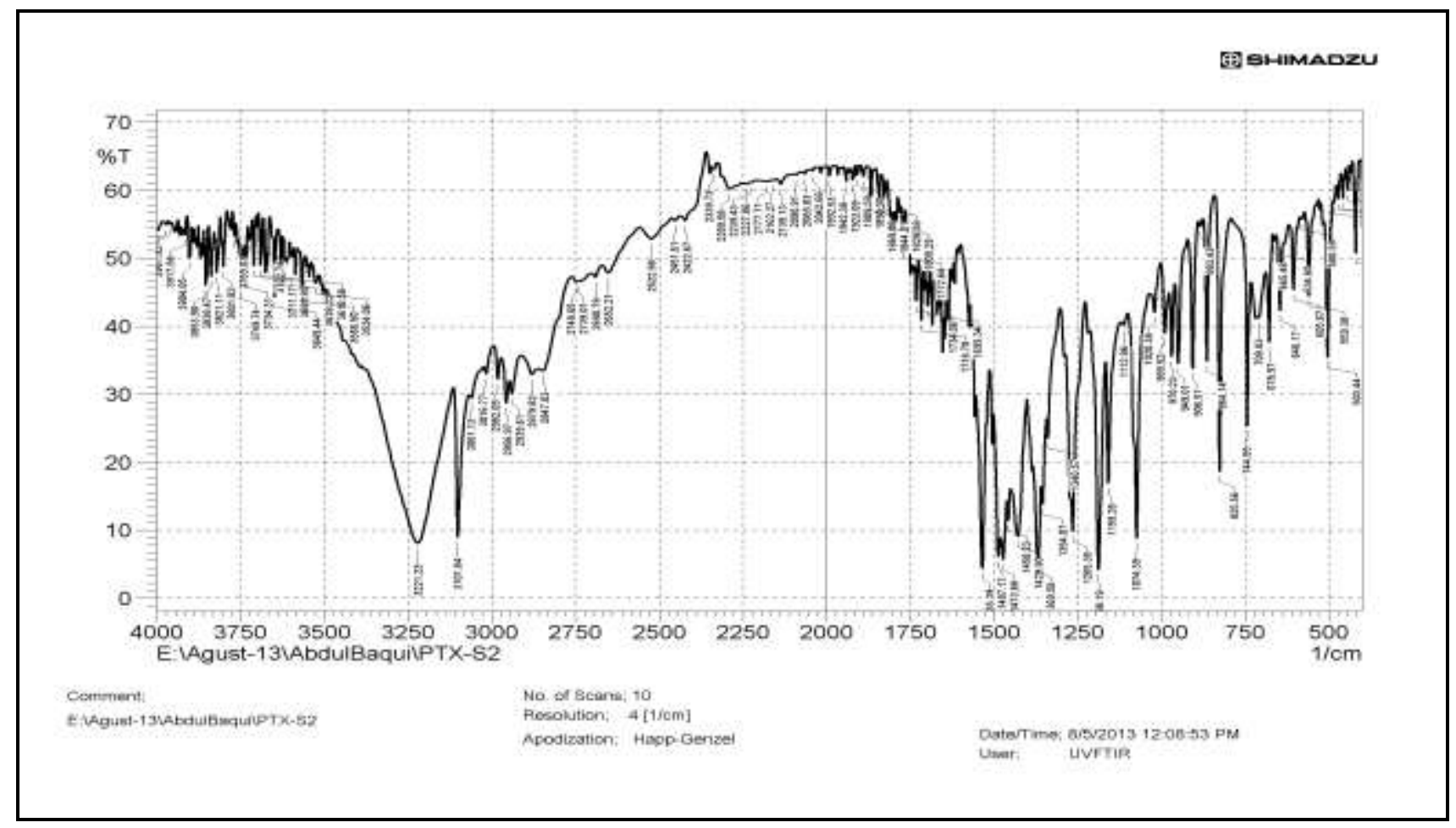

Figure no: 09 FT-IR graph of Paclitaxel-BSA+PEG6000 Nanoparticles

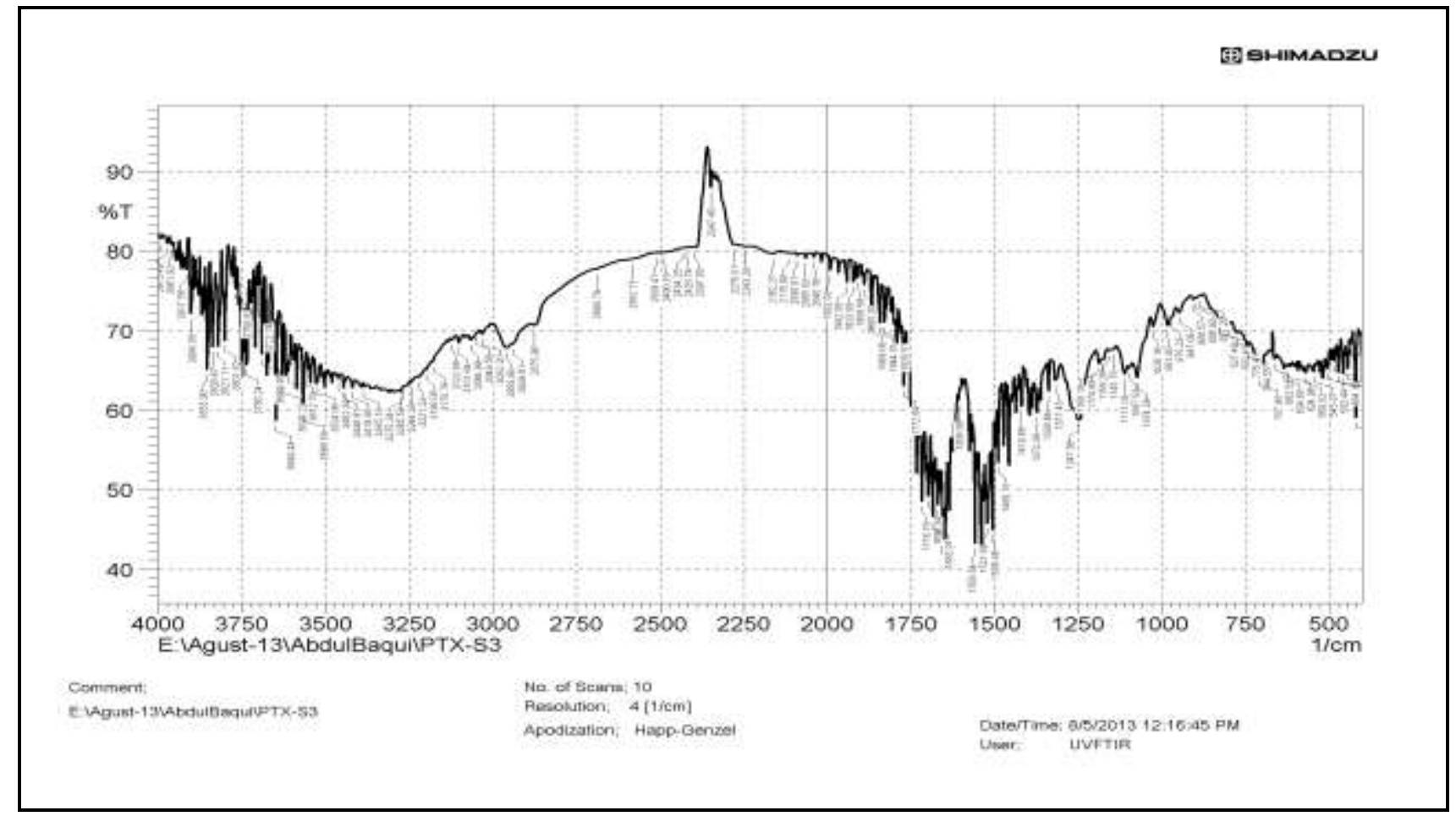

Figure no: 10 FT-IR graph of Paclitaxel-BSA+PEG4000 Nanoparticles 


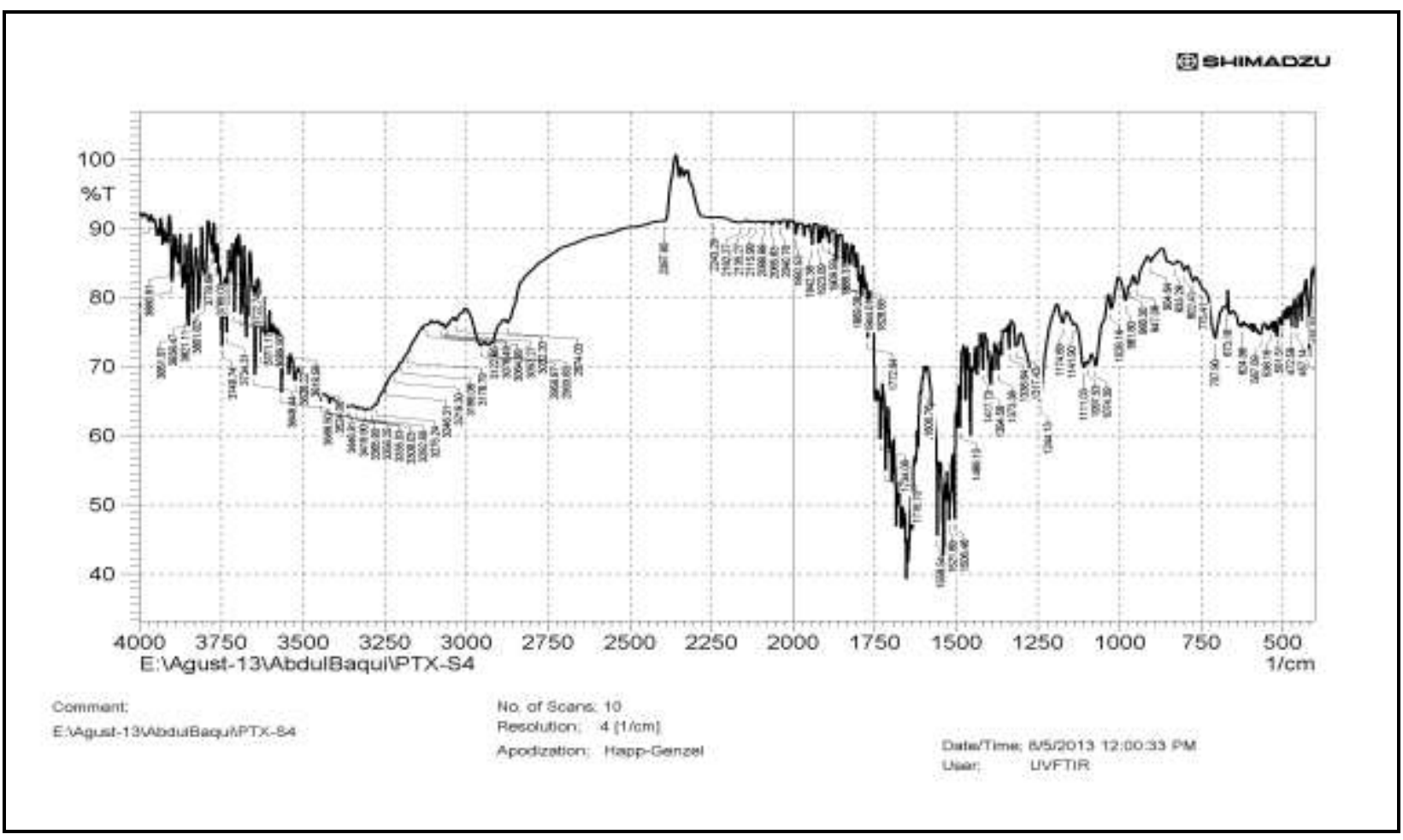

Figure no: 11 FT-IR graph of Paclitaxel-BSA+PEG1500 Nanoparticles

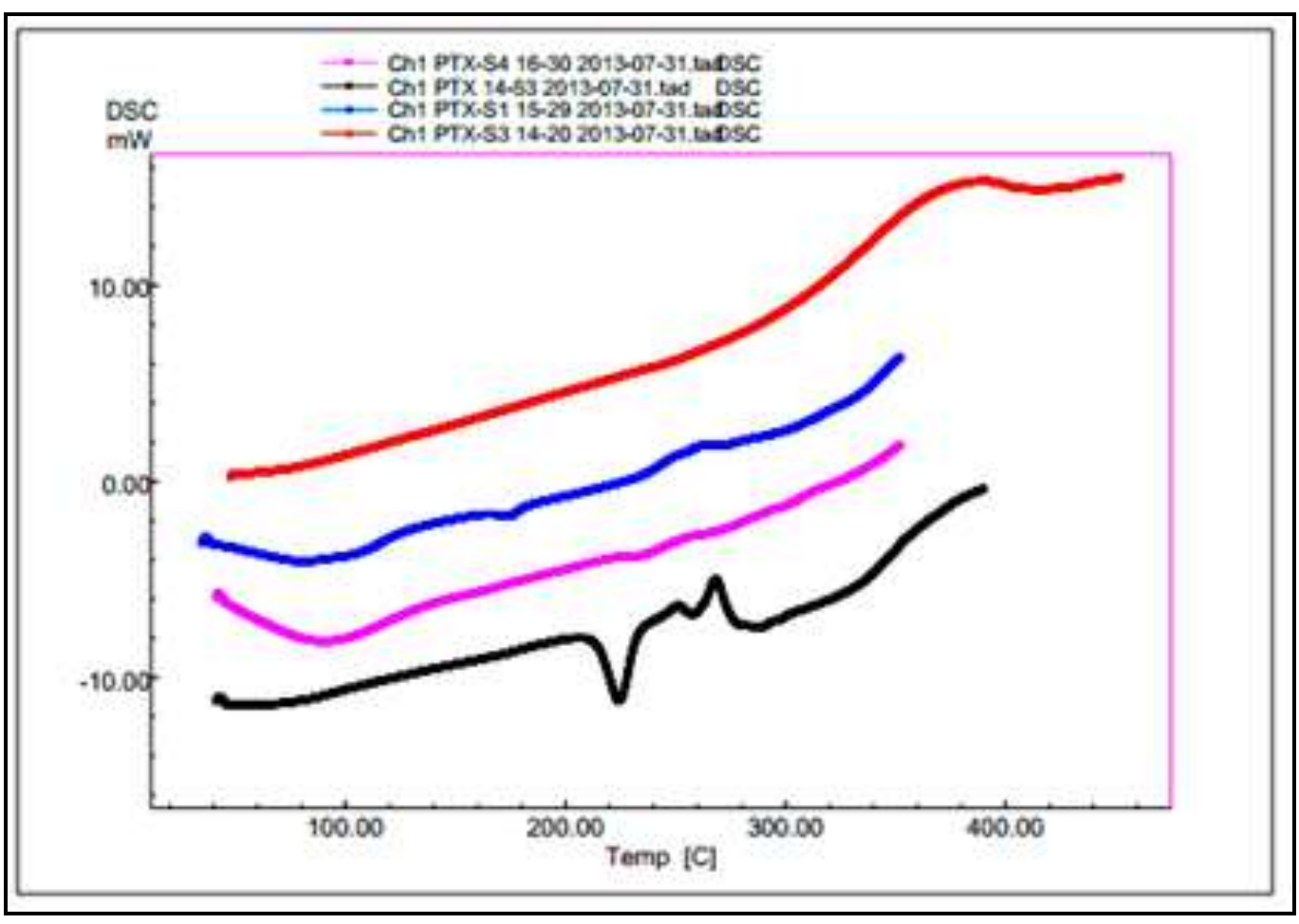

Figure no: 12 Thermograms of paclitaxel nanoparticles $S_{1}, S_{3}, S_{4}$. 


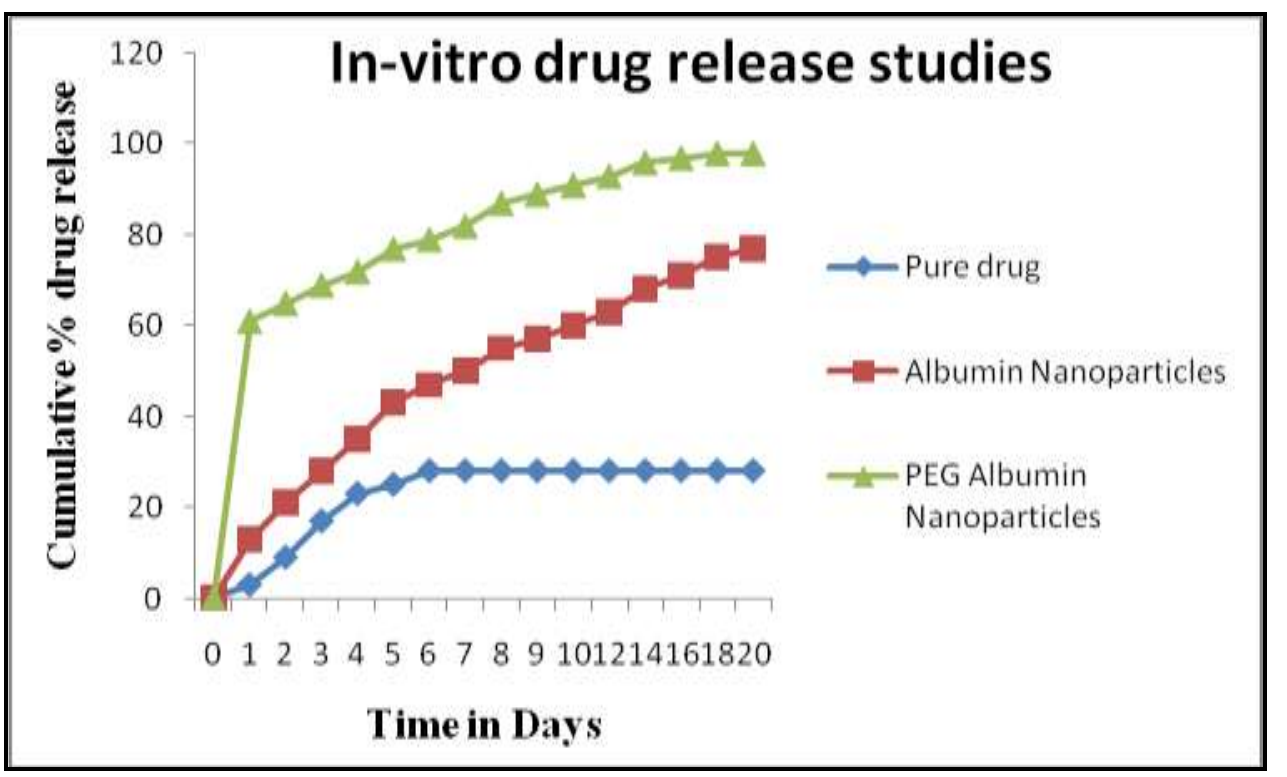

Figure no: 13 Comparative in vitro release profile of paclitaxel nanoparticles

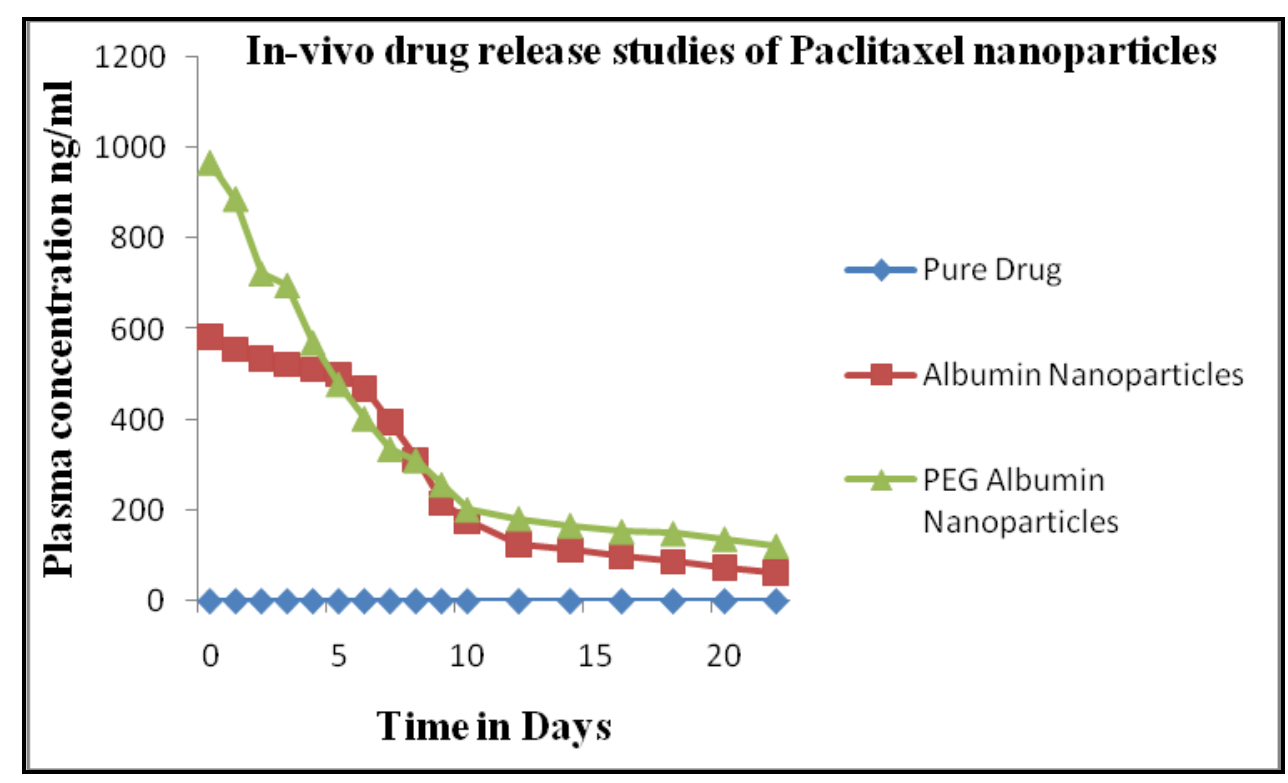

Figure no: 14 In-vivo release profiles of pure drug, albumin and pegylated nanoparticles.

\section{Conclusion:}

In conclusion, nanoparticles are one of the promising drug delivery systems, which can be of potential use in targeting drug delivery systems prepared by desolvation technique. Natural polymer like albumin can be used in the preparation. In the view of the data obtained in this study, it can also be concluded that albumin nanoparticles loaded with Paclitaxel is an effective alternatives to avoid the use of any surfactant or solubilizer (such as Tween-80) in injectable paclitaxel formulation. Our investigation suggests that albumin nanoparticles may act as a useful and safe carrier for Paclitaxel. The various uses of albumin as a drug carrier that have emerged in the past 10 years are of considerable interest and range from extending the half-life of therapeutically active proteins and peptides to drug targeting in oncology. The development and market approval of the paclitaxel albumin nanoparticle, Abraxane, can be viewed as a landmark, not only for just for albumin-based drug delivery technology but also for nanomedicine. 


\section{References:}

1. Kawasaki, E. S., \& Player, A. (2005). Nanotechnology, nanomedicine, and the development of new, effective therapies for cancer. Nanomedicine; Nanotechnology, Biology, and Medicine, 1(2), 101-109. doi:10.1016/j.nano.2005.03.002.

2. Poonthananiwatkul, B., Howard, R. L., Williamson, E. M., \& Lim, R. H. (2015). Cancer patients taking herbal medicines: A review of clinical purposes, associated factors, and perceptions of benefit or harm. Journal of Ethnopharmacology, 3, 30119-7.

3. Mirtsching B., Cosgriff T., Harker G., Keaton M., Chidiac T., Min M..: A phase II study of weekly nanoparticle albumin-bound paclitaxel with or without trastuzumab in metastatic breast cancer, Clininical Breast Cancer, 2011, (2): 121-8.

4. Hamza, A. H., Abdulfattah, H. M., Mahmoud, R. H., Khalil, W. K., \& Ahmed, H. H. (2015). Current concepts in pathophysiology and management of hepatocellular carcinoma. Acta Biochimica Polonica, 62(3), 573-580. doi:10.18388/abp.2015 1030.

5. Rajat, S., Yasir, M., \& Sanjay, B. (2011). Formulation and evaluation of Paclitaxel loaded PSA-PEG nanoparticles. Journal of Applied Pharmaceutical Science, 01(05), 96-98.

6. Sudhir S., Chakravarthi, Robinson D.H. (2011). Enhanced cellular association of paclitaxel delivered in chitosan-PLGA particles, International Journal of Pharmaceutics, 409.

7. Linhua, Z., Yingna, H., Mei, Y., \& Cunxian, S. (2011). Paclitaxel-loaded polymeric nanoparticles based on PCL-PEG-PCL: Preparation, in vitro and in vivo evaluation. Journal of Controlled Release, $\cdots$, 152.

8. Danhier, F., Lecouturier, N., Vroman, B., Jérôme, C., Marchand-Brynaert, J., Feron, O., \& Préat, V. (2009). Paclitaxel-loaded PEGylated PLGA-based nanoparticles: In vitro and in vivo evaluation. Journal of Controlled Release, 133(1), 11-17. doi:10.1016/j.jconrel.2008.09.086.

9. Agüeros, M., Ruiz-Gatón, L., Vauthier, C., Bouchemal, K., Espuelas, S., Ponchel, G., \& Irache, J. M. (2009). Combined hydroxypropyl-_-cyclodextrin and poly(anhydride) nanoparticles improve the oral permeability of paclitaxel. European Journal of Pharmaceutical Sciences, 38(4), 405-413. doi:10.1016/j.ejps.2009.09.010.

10. Xu, Z., Gu, W., Huang, J., Sui, H., Zhou, Z., Yang, Y., \& Li, Y. (2005). In vitro and in vivo evaluation of actively targetable nanoparticles for paclitaxel delivery. International Journal of Pharmaceutics, 288(2), 361-368. doi:10.1016/j.ijpharm.2004.10.009.

11. Agüeros, M., Zabaleta, V., Espuelas, S., Campanero, M. A., \& Irache, J. M. (2010). Increased oral bioavailability of paclitaxel by its encapsulation through complex formation with cyclodextrins in poly(anhydride) nanoparticles. International Journal of Pharmaceutics, 145, 2-8.

12. Lacoeuille, F., Hindre, F., Moal, F., Roux, J., Passirani, C., Couturier, O., \& Benoit, J. P. (2007). In vivo evaluation of lipid nanocapsules as a promising colloidal carrier for paclitaxel. International Journal of Pharmaceutics, 344(1-2), 143-149. doi:10.1016/j.ijpharm.2007.06.014.

13. Sahoo, S. K., \& Labhasetwar, V. (2005). Enhanced antiproliferative activity of transferrin-conjugated paclitaxel-loaded nanoparticles is mediated via sustained intracellular drug retention. Molecular Pharmaceutics, 2(5), 373-383. doi:10.1021/mp050032z.

14. Mirtsching, B., Cosgriff, T., Harker, G., Keaton, M., Chidiac, T., \& Min, M. (2011). A phase II study of weekly nanoparticle albumin-bound paclitaxel with or without trastuzumab in metastatic breast cancer. Clinical Breast Cancer, 11(2), 121-128. doi:10.1016/j.clbc.2011.03.007.

15. Jithan, A., Madhavi, K., Madhavi, M., \& Prabhakar, K. (2011). Preparation and characterization of albumin nanoparticles encapsulating curcumin intended for the treatment of breast cancer. International Journal of Pharmaceutical Investigation, 1(2), 119-125. doi:10.4103/2230-973X.82432. 